\title{
Host-plant-mediated effects of Nadefensin on herbivore and pathogen resistance in Nicotiana attenuata Cbgowda Rayapuram and Ian T Baldwin*
}

\author{
Address: Department of Molecular Ecology, Max Planck Institute for Chemical Ecology, Hans-Knöll Str. 8, D-07745 Jena, Germany \\ Email: Cbgowda Rayapuram - gowda.rayapuram@ibmp-ulp.u-strasbg.fr; Ian T Baldwin* - baldwin@ice.mpg.de \\ * Corresponding author
}

Published: 25 October 2008

BMC Plant Biology 2008, 8:109 doi:10.1 186/147|-2229-8-109
Received: 4 April 2008

Accepted: 25 October 2008

This article is available from: http://www.biomedcentral.com/I47I-2229/8//09

(c) 2008 Rayapuram and Baldwin; licensee BioMed Central Ltd.

This is an Open Access article distributed under the terms of the Creative Commons Attribution License (http://creativecommons.org/licenses/by/2.0), which permits unrestricted use, distribution, and reproduction in any medium, provided the original work is properly cited.

\begin{abstract}
Background: The adage from Shakespeare, "troubles, not as single spies, but in battalions come," holds true for Nicotiana attenuata, which is commonly attacked by both pathogens (Pseudomonas spp.) and herbivores (Manduca sexta) in its native habitats. Defense responses targeted against the pathogens can directly or indirectly influence the responses against the herbivores. Nadefensin is an effective induced defense gene against the bacterial pathogen Pseudomonas syringae pv tomato (PST DC3000), which is also elicited by attack from $M$. sexta larvae, but whether this defense protein influences $M$. sexta's growth and whether $M$. sexta-induced Nadefensin directly or indirectly influences PST DC3000 resistance are unknown.
\end{abstract}

Results: $M$. sexta larvae consumed less on WT and on Nadefensin-silenced $N$. attenuata plants that had previously been infected with PST DC3000 than on uninfected plants. WT plants infected with PST DC3000 showed enhanced resistance to PST DC3000 and decreased leaf consumption by M. sexta larvae, but larval mass gain was unaffected. PST DC3000-infected Nadefensin-silenced plants were less resistant to subsequent PST DC3000 challenge, and on these plants, M. sexta larvae consumed less and gained less mass. WT and Nadefensin-silenced plants previously damaged by $M$. sexta larvae were better able to resist subsequent PST DC3000 challenges than were undamaged plants.

Conclusion: These results demonstrate that Na-defensin directly mediates defense against PST DC3000 and indirectly against $M$. sexta in N. attenuata. In plants that were previously infected with PST DC3000, the altered leaf chemistry in PST DC3000-resistant WT plants and PST DC3000susceptible Nadefensin-silenced plants differentially reduced $M$. sexta's leaf consumption and mass gain. In plants that were previously damaged by $M$. sexta, the combined effect of the altered host plant chemistry and a broad spectrum of anti-herbivore induced metabolomic responses was more effective than Nadefensin alone in resisting PST DC3000.

\section{Background}

Plants are attacked in nature by a diverse suite of biotic challenges from pathogens and herbivores which can be devastating. But when plants are attacked by pathogens and herbivores, they mount defense responses which can slow an herbivore's feeding and also the rate of disease spread. For example, in response to herbivore attack, plants produce a broad spectrum of defense compounds that are elicited by a jasmonic acid-dependent signaling pathway. Tomato plants produce potent anti-herbivore 
defense metabolites such as proteinase inhibitors and polyphenoloxidase when attacked by Spodoptera exigua [1]. In response to damage by the solanaceous specialist herbivore Manduca sexta, Nicotiana attenuata produces anti-herbivore defense metabolites such as nicotine [2,3], caffeoyl putrescine, rutin, and diterpene glycoside [4], as well as anti-digestive trypsin protease inhibitors (TPIs) $[5,6]$. On the other hand, plants infected with fungi, bacteria or viruses produce several types of pathogenesisrelated proteins (PRs) belonging to at least 17 families [7]. Most PR proteins are known to possess antimicrobial characteristics. For instance, PR-2 (glucanases) of tobacco, barley, alfalfa and soybean have been shown to suppress diseases caused by Phytophthora megasperma f. sp. medicaginins, Rhizoctonia solani and Alternaria alternata [7]. PR-3 (chitinases) isolated from bean can suppress Rhizoctonia solani in tobacco and canola when overexpressed [8]. PR13/Na-defensins in N. attenuata have been shown to suppress Pseudomonas syrinage pvtomato-DC3000 (PST DC3000) [9].

These examples clearly suggest that depending on the type of the attacker, plants can produce different blends of defense metabolites. But in nature, plants often have to deal with not one but several natural enemies, and these can occur either simultaneously or one after the other, with one enemy facilitating or eliciting resistance to the attack of subsequent attackers. Insects are also wellknown vectors for pathogens [10]; moreover, attack by rust fungi can influence subsequent herbivory in the same plant species [11]. The co-evolution of plants and their natural enemies makes it likely that plant responses related to one attacker have far-reaching consequences for subsequent attackers. Plants often produce secondary metabolites with generalized detrimental effects on herbivores as well as pathogens. The phenolic compound rutin is such an example [12]. Constitutive levels of iridoid glycosides among natural variants of Plantago lanceolata have been shown to confer resistance to the herbivore Spodoptera exigua as well as to the biotropic fungal pathogen Diaporthe adunca [11]. Recently it was shown that caterpillar feeding significantly reduced the extent of disease caused by the bacterial pathogens PST DC3000 and Xanthomonas campestris pvarmoraciae in Arabidopsis [13].

Although many studies have shown that pathogen- and herbivore-elicited plant responses can have detrimental effects on subsequent attacker(s), few have established causal associations with the induction of a specific metabolite(s) by the first attacker with a detrimental effect (direct or indirect) on subsequent attacker(s). Some studies have causally linked the signaling pathways that mediate effects against herbivores and pathogens [13], others have found associations among the amounts of constitutively expressed metabolites (iridoid glycosides) known to affect both herbivores and pathogens; but all these studies have been conducted with genotypes selected over generations for high- and low-leaf iridoid glycoside concentrations [11] and are not iso-genic. Therefore, they may differ in resistance traits other than the measured metabolites. As with constitutively produced metabolites, little is known about the cross effects of inducible defense metabolites.

Here we 1) identify a defense metabolite (Na-defensin) in WT N. attenuata that is elicited in response to attack from both pathogens and herbivores and 2) examine the consequences of this metabolite for both pathogen and herbivore resistance by comparing the resistance of WT and isogenic plants transformed to silence the expression of the metabolite. N. attenuata, a solanaceous annual originating from the Great Basin Desert or North America, dramatically increases Nadefensin (also known as PR-13) levels when attacked by herbivores such as Manduca sexta larvae $[14,15]$, Tupiocorus notatus, Myzus nicotianae, Spodoptera littoralis and Trichoplusia ni [16] as well as the pathogen PST DC3000 [9]. Previous work from our lab has shown that WT N. attenuata plants silenced for Nadefensin made WT plants increasingly susceptible to PST DC3000 [9]. Our main objectives were to study 1) the role of defensin in $N$. attenuata's induced resistance to M. sexta and 2) the influence of defensin on M. sexta growth when elicited by PST DC3000 and vice versa. We began by observing the patterns of Nadefensin expression in $N$. attenuata in response to M. sexta and PST DC3000 and then studied the effect of Nadefensin on M. sexta's resistance to plants that had been previously infected with PST DC3000 as well as the effects of Nadefensin on PST DC3000 resistance when plants had been previously damaged by M. sexta.

\section{Results \\ Gene expression analysis of Nadefensin in WT and transgenic N. attenuata plants silenced for Nadefensin}

In N. attenuata, Nadefensin (NCBI accession AY456268) is up-regulated in WT N. attenuata plants after attack from M. sexta [14,15], Tupiocorus notatus, Myzus nicotianae, Spodoptera littoralis and Trichoplusia ni [16] larvae. Bacteria (Pseudomonas syringae) are also known to induce defensin in different plants $[17,18]$. Recently, we reported that Nadefensin was up-regulated $12 \mathrm{~h}$ after WT N. attenuata plants were infected with PST DC3000 [9]; moreover, silencing Nadefensin by RNAi by stable transformation (irdefensin lines 76 and 96) increased the plant's susceptibility to PST DC3000 [9].

While attack from both M. sexta larvae and PST DC3000 is known to elicit Nadefensin transcripts and protein in $\mathrm{N}$. attenuata, the relative responses to M. sexta larvae and PST DC3000 challenges were not known. We re-examined the levels of Nadefensin transcripts accumulation in PST 
DC3000 and $M$. sexta-attacked plants at a single time point (4 days after pathogen and herbivore damage). The quantitative real-time PCR (qRT-PCR) analysis (Fig. 1) revealed that Nadefensin transcript accumulation differed significantly across treatments and genotypes (Fig. 1; ANOVA $\left.F_{11,17}=16.00, P<0.001\right)$ : Nadefensin levels in WT plants infected with PST DC3000 and in those attacked by M. sexta did not differ significantly (Fig. $1 ; p=0.183$ ). Consistent with our earlier observation [9], WT plants either damaged by M. sexta or infected by PST DC3000 had significantly more (at least 60\%) Nadefensin transcripts compared to similarly treated irdefensin plants (76 and 96). The similar levels of Nadefensin after M. sexta damage or PST DC3000 infection suggest that Nadefensin is likely elicited by jasmonates which are produced in response to pathogen infection as well as insect attack [19].

\section{Effects of PST DC3000 infection and Nadefensin silencing on herbivore performance}

Since Nadefensin is expressed in response to attack from both herbivores and pathogens, we asked if silencing Nadefensin expression influenced $M$. sexta growth in uninduced plants as well as in plants previously inoculated with PST DC3000. We carried out assays on WT and Nadefensin-silenced plants (lines 76 and 96) which were either uninduced or had been infected (4 days earlier) with PST DC3000. We measured two parameters that reflect the

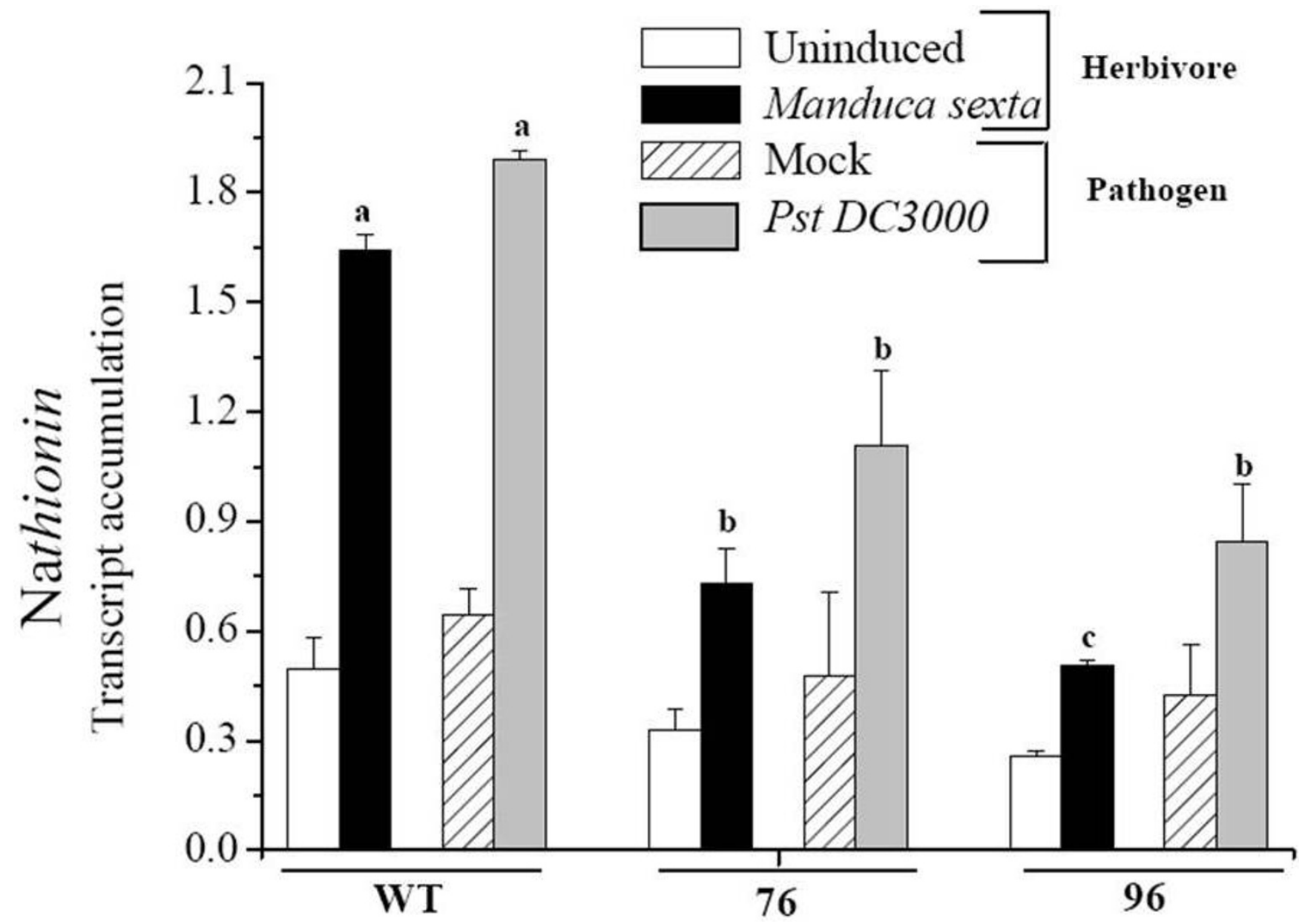

Figure I

M. sexta damage and Pst DC3000 inoculation increase Nadefensin transcripts; responses are highly attenuated in irdefensin (76 and 96) lines. Quantitative real-time PCR (qRT-PCR) was used to analyze Nadefensin transcript accumulation in WT N. attenuata plants and irdefensin (76 and 96) lines in response to continuous $M$. sexta feeding by first-instar larvae for 4 days or inoculation with Pseudomonas syringae pv tomato DC3000 (Pst DC3000) $\left(\mathrm{I} \times 10^{5} \mathrm{cells} / \mathrm{ml}\right)$. Values are mean $( \pm \mathrm{SE})$ Nadefensin transcripts from 3 replicate plants per treatment normalized to the transcript abundance of actin, which is unregulated under these conditions. Different letters indicate significant differences between genotypes damaged by $M$. sexta and infected by Pst DC3000. 
overall performance of $M$. sexta larvae: percentage of leaf area damage and larval mass gain.

\section{Percentage of leaf area damage}

After 12 days of attack from a single $M$. sexta, leaves were evaluated for the percentage of leaf area damaged. M. sexta larvae removed significantly more leaf area from uninduced WT and irdefensin (76 and 96) plants (at least 30\%) than from PST DC3000-infected plants (Fig. 2A and 2B; ANOVA, $\left.F_{5,88}=19.67, P<0.001\right)$. Within the uninduced treatment, no significant differences in the percentage of leaf area damage between WT and irdefensin line $76(p=$ $0.905)$ plants or between WT and irdefensin line $96(p=$ 0.517) plants were observed (Fig. 2A). On the other hand, prior infection with PST DC3000 resulted in greater leaf area losses (at least 35\%) in WT plants compared to plants from both irdefensin lines (Fig. 2A and 2B; line $76 p<$ 0.001 ; line $96 p=0.002$ ).

\section{Larval mass gain}

We also measured the mass of the larvae that fed on uninduced and on PST DC3000-infected WT and irdefensin (76 and 96) plants. ANOVA revealed significant differences among the treatments and the genotypes (Fig. 3A and 3B; ANOVA, $\left.F_{17,426}=14.14, P<0.001\right)$, but the larval mass differences differed from those of the pattern leaf area damaged. No significant differences in larval mass between the M. sexta larvae that fed on the uninduced WT and those that fed on WT plants which were PST DC3000 infected was observed $(p=0.264)$. Within the uninduced treatment, no statistical differences in the mass of larvae that fed on WT and irdefensin line $76(p=0.427)$ plants or WT and irdefensin line $96(p=0.117)$ plants were observed (Fig. 3A). On the other hand, larvae that fed on WT plants infected with PST DC3000 gained significantly more (at least $70 \%$ ) mass than did larvae that fed on infected plants from irdefensin line $76(\mathrm{p}=0.012)$ and irdefensin line 96 ( $\mathrm{p}=0.045$ ). The larvae that fed on PST DC3000-infected irdefensin (76 and 96) plants were smaller than the larvae that fed on PST DC3000-infected WT plants (Fig. 3B). The large variation in the larval mass across the experiment could be attributed to the differences in larvae's development which in turn may be attributed to high spatial heterogeneity in food quality for the larvae consuming infected leaves.

\section{Detecting PST DC3000 from infected plants in herbivores' guts}

In our earlier work, we reported that irdefensin (76 and 96) plants were more susceptible to PST DC3000 than were WT N. attenuata plants, and as a result irdefensin (76 and 96) plants contained more PST DC3000 colony forming units (CFUs) than did the WT plants [9]. In this study we observed that M. sexta larvae that fed on irdefensin (76 and 96) plants were smaller and seemed to be infected with pathogens (Fig. 3B). Therefore, we hypothesized that herbivores feeding on PST DC3000-infected irdefensin (76 and 96) plants might have ingested more PST DC3000 than did the larvae feeding on the PST DC3000-infected WT plants, and that the number of ingested PST DC3000 might negatively correlate with larval growth. We counted the CFUs of plant-derived PST DC3000 in guts (including the foregut, midgut and hindgut) of larvae that fed either on PST DC3000-infected WT plants or PST DC3000infected irdefensin plants (76 and 96) (Fig. 4A and 4B). As expected, we found PST DC3000 colonies in larvae that fed on PST DC3000-infected WT and irdefensin (76 and 96) but none in larvae that fed on uninfected WT and irdefensin (76 and 96) plants. However, the number of PST DC3000 colonies in the guts of larvae that fed on PST DC3000-infected WT or irdefensin (76 and 96) plants did not differ significantly (Fig. 4A; ANOVA, $F_{5,24}=2.07, P=$ 0.104 ). Moreover, the overall number of CFUs was very low relative to the number of CFUs found in leaves, which suggests that plants infected with PST DC3000 do not detrimentally affect larvae by directly transmitting pathogens to the herbivores. In addition to PST DC3000, we also detected a few unknown microorganisms with resistance to tetracycline and rifamycin (the selection markers for PST DC3000). Interestingly these unknown microorganisms were found most often in guts extracted from larvae that fed on irdefensin (76 and 96) plants. irdefensin (76 and 96) plants also show an increased susceptibility to the many opportunistic microorganisms which may be detrimental to larvae as well (Fig. 4B).

\section{Effects of herbivory and Nadefensin silencing on PST DC3000 infection}

Silencing Nadefensin expression in $N$. attenuata does not influence the plant's resistance to M. sexta attack but lowers resistance to PST DC3000 [9], which suggests that $\mathrm{Nadefensin}$ functions as an antibacterial defense protein in $N$. attenuata. We therefore explored whether Nadefensin still functions as an antibacterial protein in leaves that are damaged by herbivores. We compared the level of disease progression of PST DC3000 in leaves that were either undamaged or previously damaged (4 days) by M. sexta. Two and four days after PST DC3000 infection, leaves were evaluated for CFUs. In general, we found that inoculating leaves of undamaged plants with PST DC3000 or infecting leaves of $M$. sexta-damaged (4 days of feeding) plants with PST DC3000 resulted in statistically significant differences in PST DC3000 growth responses in N. attenuata (Fig. 5; ANOVA, $F_{17,72}=128.75, P<0.001$ ). Investigating the genotypic and treatment effects, we found the following patterns on day 4: 1) PST DC3000 CFUs were higher in both uninduced irdefensin line 76 $(9 \% ; p=0.031)$ and line $96(6.6 \% ; p=0.047)$ than in uninduced WT plants; 2) similarly, PST DC3000 CFUs were higher in M. sexta-damaged plants from both irde- 
A

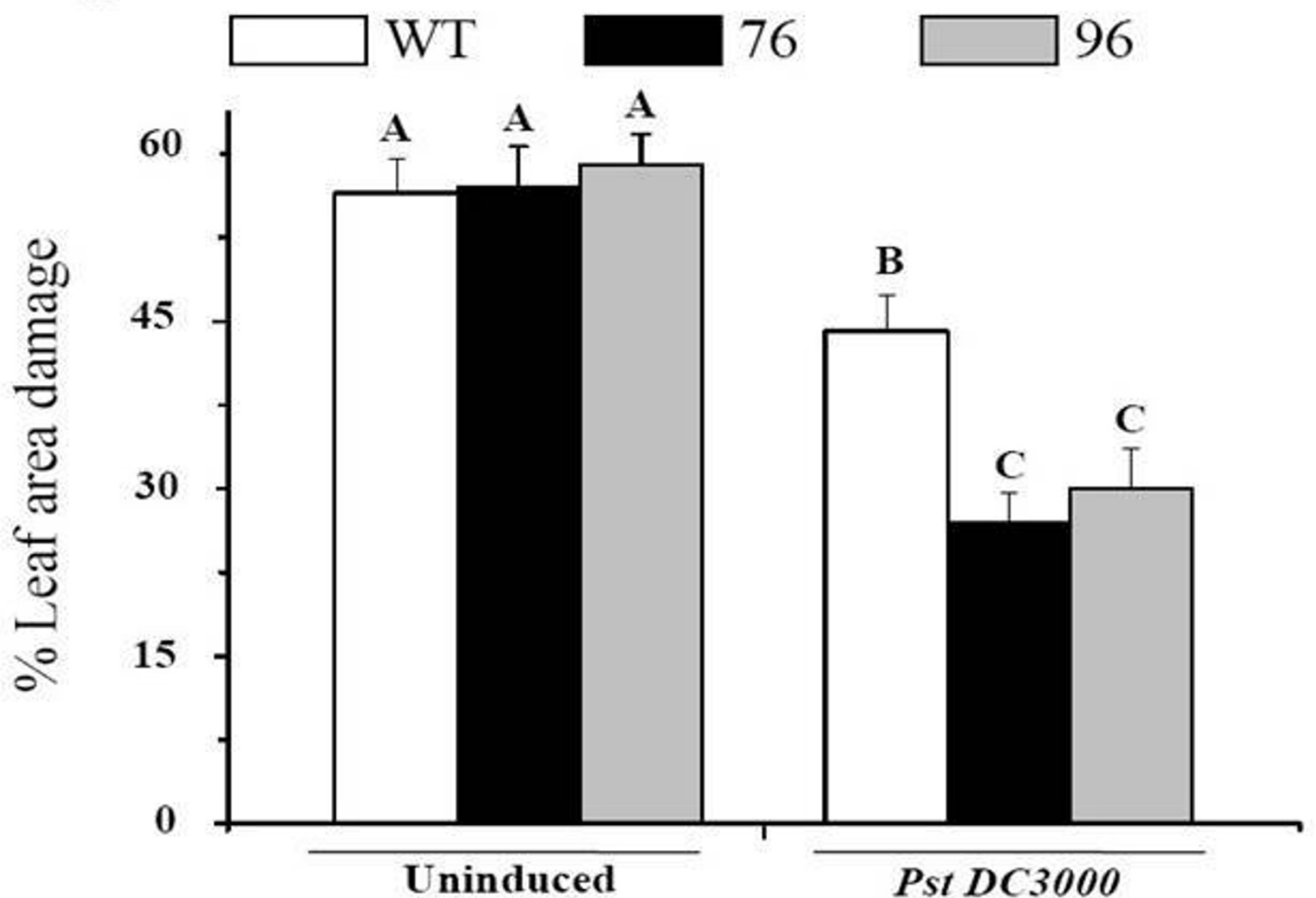

B

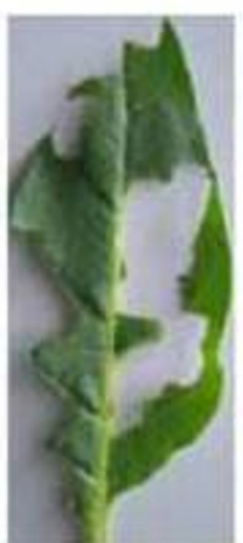

WT

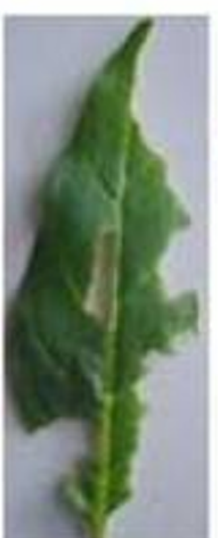

76

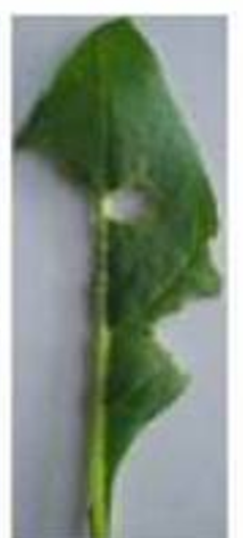

96

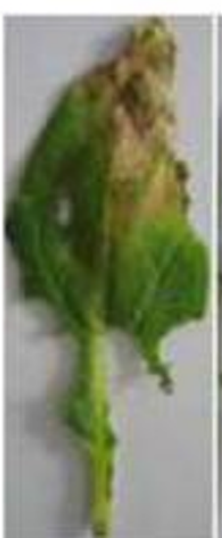

WT

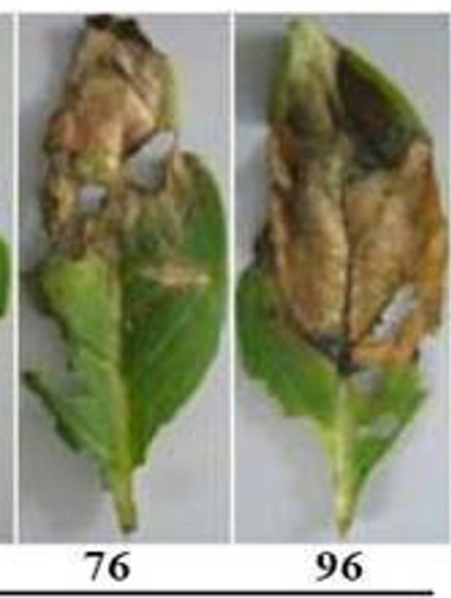

Pst DC3000

Figure 2

Pst DC3000 inoculation and Nadefensin silencing decreases leaf area damage by $M$. sexta larvae in N. attenuata. A) Mean $( \pm$ SE) percentage of leaf area damage by $M$. sexta larvae on WT plants and irdefensin lines 76 and 96 . A neonate larva was placed in a clip cage and allowed to feed for 12 days before the percentage of leaf area damage was estimated. B) Photographs taken after 12 days of M. sexta feeding on WT and irdefensin lines 76 and 96 that were either uninduced (left) or inoculated with Pst DC3000 (right). Different letters indicate significant differences between treatments and genotypes $(\mathrm{N}=18)$. 


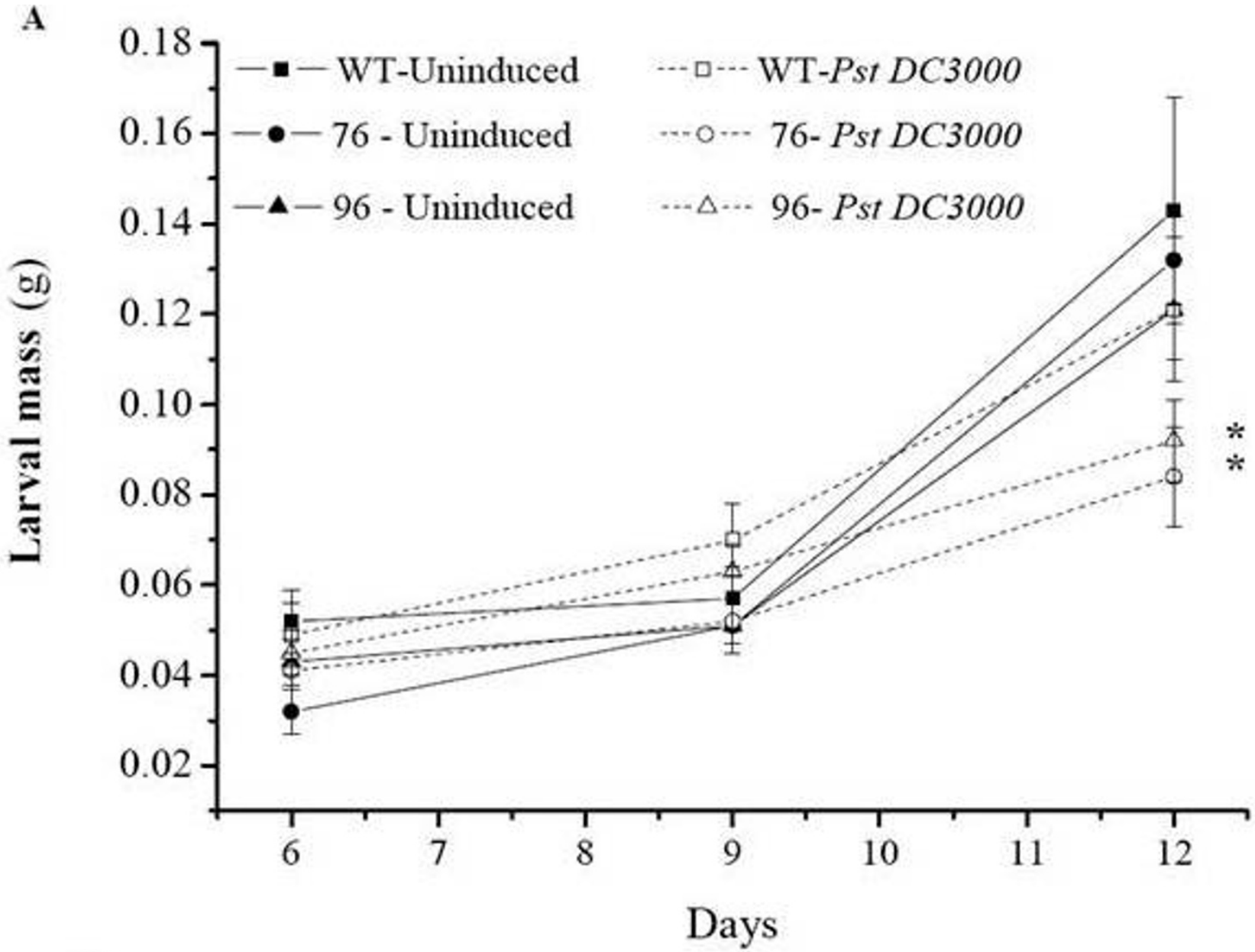

B

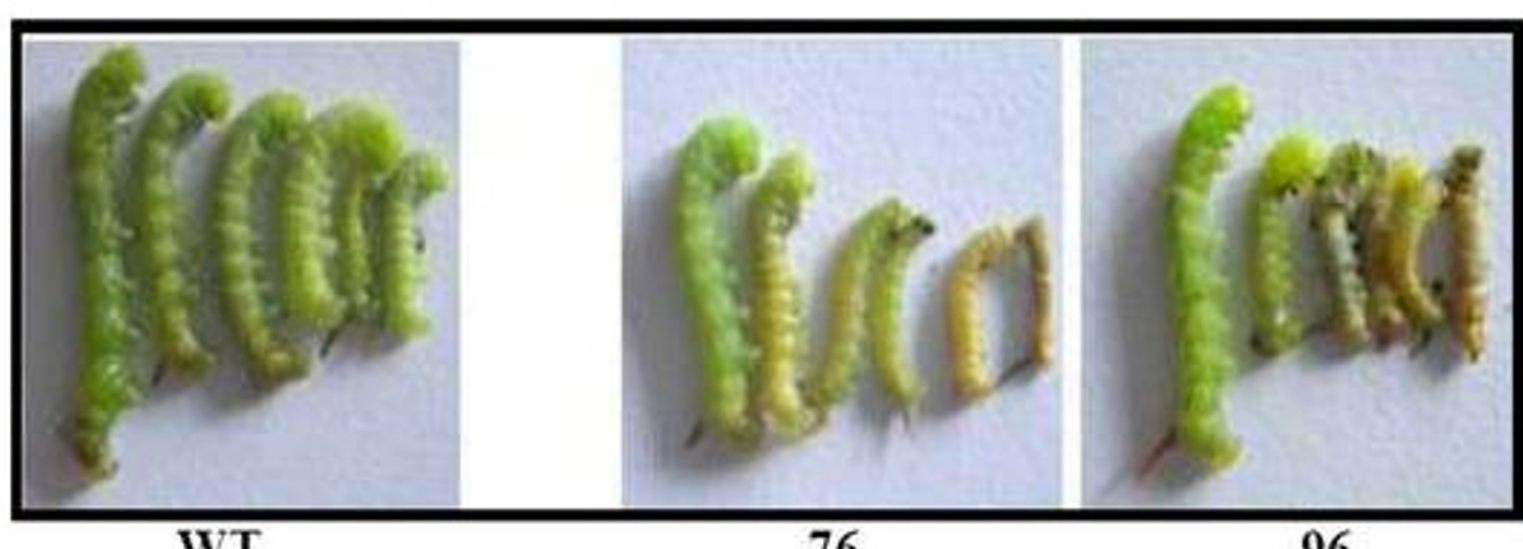

Figure 3

Pst DC3000 inoculation and Nadefensin silencing decrease $M$. sexta larval mass gain in $\mathbf{N}$. attenuata. A). Mean ( \pm SE) M. sexta larval mass gain on WT plants and irdefensin lines 76 and 96 . A neonate larva was placed in a clip cage and allowed to feed continuously for 12 days. Larval mass was recorded on days 6, 9 and I2. B) Photographs taken after 12 days of $M$. sexta feeding on WT and irdefensin lines 76 and 96 that were induced with Pst DC3000. Asterisk indicates significant differences $(p=$ $0.05)$ between WT and irdefensin lines (76 and 96) after Pst DC3000 infection $(N=30)$. 

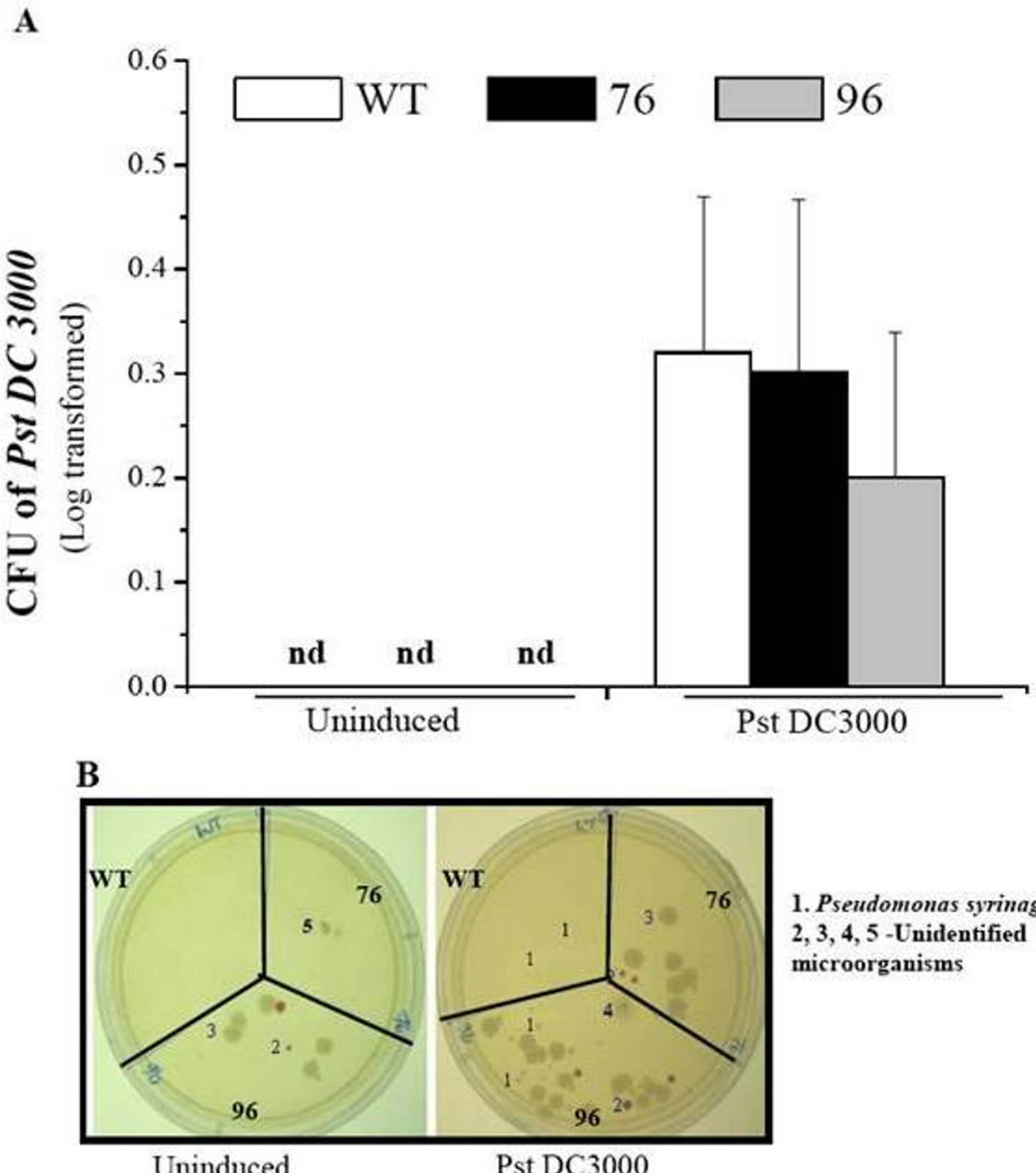

1. Pseudomonas syrinage Pst DC 3000 2, 3, 4, 5 -Unidentified microorganisms

Figure 4

The number of Pst DC3000 colonies quantified in the guts of $M$. sexta larvae that fed on the Pst DC3000-inoculated WT and irdefensin (76 and 96) plants do not differ. A) Mean ( \pm SE) colony-forming units (CFUs) of Pst DC3000 in the guts of larvae that fed on either the Pst DC3000-inoculated WT/irdefensin (76 and 96) or uninduced WT/irdefensin (76 and 96) plants. The larval guts from 5 replicate larvae that fed on either Pst DC3000-inoculated WT and irdefensin (76 and 96) for 12 days were surgically removed and ground in I $\mathrm{ml}$ sterile water. $40 \mu \mathrm{l}$ of supernatant was spread on plates containing LB agar plate containing rifamycin and tetracycline to select for the growth of Pst DC3000. Colonies were counted after $48 \mathrm{~h}$ of incubation at $28^{\circ} \mathrm{C}$. B) Photographs of LB plates + antibiotics (rifamycin and tetracycline) showing Pst DC 3000 , in addition to four unidentified/unknown microorganisms that could also grow on LB plants supplemented with antibiotics $(\mathrm{N}=5)$. 


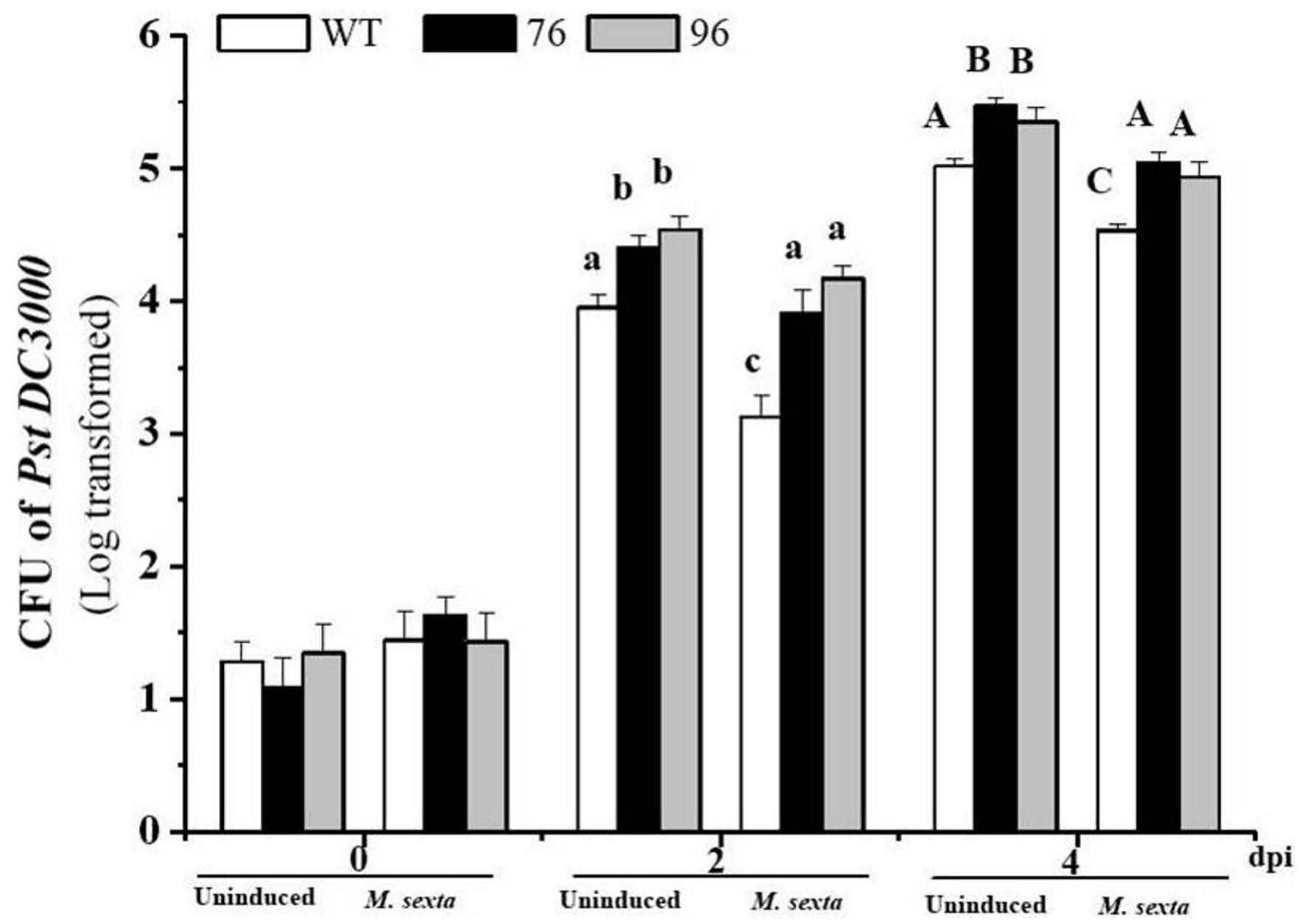

Figure 5

M. sexta feeding significantly reduces Pst DC 3000 disease spread. Values are mean ( \pm SE) colony-forming units (CFUs) of Pst DC3000 after inoculation of the leaves of WT/irdefensin (76 and 96) plants that were either uninduced or previously attacked by $M$. sexta larvae 4 days earlier. To record the CFUs, surface-sterilized leaf discs $\left(\mathrm{I} \mathrm{cm}^{2}\right)$ were ground in I ml sterile water and $40 \mu \mathrm{l}$ of supernatant was spread on plates containing LB agar + antibiotics (rifamycin and tetracycline). Colonies were counted after $48 \mathrm{~h}$ of incubation at $28^{\circ} \mathrm{C}$. Different letters in lower and upper cases indicate significant differences among Pst DC3000-inoculated WT plants and the transgenic plants on days 2 and day 4, respectively $(\mathrm{N}=5)$.

fensin lines $76(11.3 \% ; p=0.014)$ and line $96(8.9 \% ; p=$ 0.048 ) than in WT plants; 3 ) within the WT plants, control plants (undamaged) had higher titers of PST DC3000 CFUs (10.6\%; $p=0.017)$ than did $M$. sexta-damaged plants; 4) within the irdefensin lines (76 and 96) the same effects of Manduca damage were observed: control plants (undamaged) had a higher titers of PST DC3000 CFUs, $8.53 \%(p=0.037)$ and $8.51 \%(p=0.0183)$, respectively, than did the $M$. sexta-damaged lines; 5 ) control WT plants (T4-undamaged) and M. sexta-damaged irdefensin lines 76 and 96 did not differ in PST DC3000 CFUs $(p=0.934$ and $p=0.676$, respectively). In summary, these results suggest that Na-defensin's anti-bacterial defense property is retained in leaves regardless of whether a leaf is elicited by pathogen or herbivore. In addition, M. sexta damage which results in the elicitation of a large set of anti-herbivory defense metabolites more effectively restricted PST DC3000 growth than did elicitation by Na-defensin alone.

\section{Discussion}

Two observations motivated us to study the direct and indirect roles of Nadefensin in resistance to pathogens and herbivores in $N$. attenuata: 1) Nadefensin levels are increased in $N$. attenuata in response to attack from these two natural enemies and 2) Nadefensin is effective in resisting PST DC3000 in N. attenuata but not herbivores [9]. Therefore we asked: Does Nadefensin (an anti-bacte- 
rial defense gene) have a function during herbivore attack? This question led us to ask if Nadefensin indirectly affects interactions between M. sexta and PST DC3000.

The results demonstrate that PST DC3000-infection significantly reduces $M$. sexta's leaf consumption and growth; its effects are most dramatically seen in the Nadefensinsilenced plants. Since Nadefensin-silenced plants are more susceptible than WT plants to PST DC3000 [9], we inferred that either the presence of PST DC3000 in the larval diet or the altered leaf chemistry resulting from PST DC3000 infection in Nadefensin-silenced plants was responsible for the poor larval performance. Larvae that fed on PST DC3000-infected WT plants, which are resistant to PST DC3000, consumed significantly more than those that fed on PST DC3000-infected Nadefensinsilenced plants, which are highly susceptible to PST DC3000 (Fig. 2A and 2B); however, we found no difference in the titers of PST DC3000 in the guts of the larvae (Fig. 4A and 4B) that had consumed either WT or Nadefensin-silenced plants. We propose that changes in plant chemistry associated with differences in PST DC3000 resistance negatively affect leaf consumption, but not the pathogen per se. This scenario is consistent with earlier studies in which tomato leaflets that had an increase in polyphenol oxidase after being infected by PST DC3000 decreased the suitability of non-inoculated leaflets of the same leaf for Helicoverpa zea [1]. Interestingly, our results show that despite differences in leaf consumption between $M$. sexta that fed on either uninduced WT or Nadefensin-silenced plants and those that fed on PST DC3000-infected WT plants, larvae nevertheless gained the same amount of body mass (Fig. 3A). Since body mass is maintained at lower levels of consumption, PST DC3000 infection of WT plants appears to increase the efficiency by which ingested food creates body mass for M. sexta larvae.

We noticed that larvae avoided consuming the portions of the leaf exhibiting disease symptoms (Fig. 2B) and found leaf consumption to be inversely related to the size of the disease lesions. Why do larvae feeding on PST DC3000infected WT plants gain more mass even when they consume less? Plant responses to pathogen infection such as increased salicylic acid (SA), which is produced in response to PST DC3000 infection, could be involved [9]. Several studies have shown that growth in herbivores is enhanced in plants that have elevated SA levels because SA can antagonize the oxylipin signaling which mediates herbivore resistance [20-22]. However, WT and Nadefensinsilenced plants infected by PST DC3000 have the same level of SA [9], so other unmeasured changes in leaf chemistry are likely involved.

Nadefensin can function as an anti-bacterial protein and is capable of inhibiting PST DC3000 growth. Since Nade- fensin is also induced after M. sexta damage [14,15], we hypothesized that after herbivore damage Nadefensinsilenced plants should also be susceptible to PST DC3000. Indeed, Nadefensin-silenced plants that were uninduced or previously damaged by $M$. sexta were more susceptible to PST DC3000 than the WT plants that were uninduced or previously damaged by M. sexta, respectively. Clearly, $\mathrm{Na}$-defensin functions as an anti-bacterial defense protein regardless of whether it is elicited by either pathogens or herbivores. Moreover, it appears that herbivore-mediated cellular changes do not affect Nadefensin's known antibacterial function. This is not surprising given the structure of the protein. Nadefensin belongs to the $\gamma$-Nadefensin class, which is $40-45$ amino acids long, with 8 conserved cysteine residues that form 4 disulphide bridges; these disulphide bridges are thought to contribute to the stability of the protein. Moreover, Na-defensin is a cationic-basic protein and thus can be attracted to bacterial or fungal cell membranes, where it interacts directly [23]. We do not know exactly what feature of plant responses (other than jasmonates) towards pathogens or herbivores causes the induction of Nadefensin, but plants may elicit Nadefensin to prime their defense responses to possible bacterial damage. The wounds that herbivore feeding causes are likely entry points for many pathogens. This scenario is consistent with the lower PST DC3000 growth in WT or Nadefensin-silenced plants that were previously fed on by M. sexta compared to WT or Nadefensinsilenced plants that were previously undamaged. M. sextadamaged plants had reduced PST DC3000 CFUs, which is consistent with other studies reporting that herbivoredamaged plants may be less suitable for pathogens $[24,25]$. Herbivore damage is associated with the production of several defense metabolites that can have detrimental effects on herbivores as well as pathogens. For instance, $M$. sexta damage increases nicotine production in N. sylvestris[26] and N. attenuata [3], and nicotine was found to inhibit the growth of five species of Pseudomonas bacterial pathogens [12]. Similarly, many phenolic compounds such as rutin and chlorogenic acid are also produced in N. attenuata [4]. Rutin, for instance, is a broad spectrum defense metabolite [12]. Accordingly, after herbivory plants likely elicit herbivore-specific metabolites as well as anti-bacterial defense proteins such as defensin. The latter may be elicited in anticipation that the wounds created during herbivory might provide an opening for pathogenic bacteria.

\section{Conclusion}

We studied the three-way interaction between a plant $(N$. attenuata), an herbivore ( $M$. sexta) and pathogenic bacteria (PST DC3000) with reference to a known anti-bacterial defense protein, Na-defensin. Na-defensin was found to be effective in containing PST DC3000 growth but ineffective in preventing M. sexta from feeding and gaining body mass. When the indirect effects of Nadefensin on herbivore 
performance were analyzed in PST DC3000-infected WT leaves, we found that the increased expression of Nadefensin increased resistance to PST DC3000 and also reduced $M$. sexta's leaf consumption but not its larval mass gain. Reduced Nadefensin expression in PST DC3000infected Nadefensin-silenced plants decreased resistance to PST DC3000 but hindered M. sexta's leaf consumption and its mass gain. When we analyzed the Nadefensinmediated responses in $M$. sexta-damaged leaves to PST DC3000 resistance, we found that damage by M. sexta increased the resistance of both WT and Nadefensinsilenced plants to PST DC3000. This enhanced resistance in M. sexta-damaged plants may result from the elicitation of a large set of anti-herbivory defense metabolites that can affect both herbivores as well as pathogens and to some specific anti-bacterial defense proteins such as defensin.

\section{Methods \\ Plant material, Pseudomonas syringae growth, plant treatments}

Wild-type (WT) N. attenuata plants (seeds collected from a native population from the DI Ranch, Santa Clara, UT, USA) that had been inbred for 14 generations were used in this study. Transgenic plants, irdefensin lines (76 and 96), were produced in the same WT genetic background. Germination was carried out according to the procedures described in [27]. Plants were grown in chambers (16/8 hr photoperiod at $25 / 21^{\circ} \mathrm{C}$, and $45-55 \%$ relative humidity) and experiments were carried out with rosette-stage plants 14 days after they were transferred to $1 \mathrm{l}$ pots.

The virulent strain of Pseudomonas syringae pv tomatoDC3000 (PST DC3000) strain was grown and maintained on a LB agar plates at $28^{\circ} \mathrm{C}$. The PST DC3000 growth and inoculation procedure was carried out as described in [28]. In brief, $1 \times 10^{5}$ cells $/ \mathrm{ml}$ were resuspended in $0.1 \%$ Silwett L-77 solution and intact leaves were dipped for 1 minute. As a mock inoculation, leaves were dipped in $0.1 \%$ Silwett L-77 solution.

\section{Isolating Nadefensin, and generating and characterizing Nadefensin-silenced plants}

Nadefensin was identified as a differentially regulated gene in WT N. attenuata plants that had been damaged by the specialist herbivore $M$. sexta. The sequence has been submitted in the NCBI database (accession number AY456268) [14]. Using a forward primer (FLTIO-FP: 5'ATGGCTCGATCCTTGTGCTTCATG 3 ' and a reverse primer FLTIO-RP: 5'TTAGTTATCCATCATCTCTTC 3'), an Nadefensin sequence was PCR amplified from the cDNA obtained from PST DC3000-inoculated WT leaves and this sequence was used to generate transgenic plants irdefensin lines (76 and 96) as described in [9]. In brief, a 225 bp fragment from Nadefensin ORF was inserted into a
pRESC5 transformation vector to create an inverted-repeat (ir) construct. These constructs were transformed into $\mathrm{N}$. attenuata WT plants using an Agrobacterium-mediated transformation procedure described in [27]. The gene for hygromycin resistance ( $h p t I I$ ) allowed transformed plants to be identified easily by selecting hygromycin-resistant individuals [27].

\section{Nucleic acid analysis \\ Transcript analysis}

To analyze Nadefensin transcripts, we extracted total RNA with TRIzol reagent (Invitrogen, http://www.invitro gen.com) following the TIGR protocol http:// www.tigr.org). cDNA was synthesized from $1 \mu \mathrm{g}$ RNA using the SuperScript ${ }^{\mathrm{TM}}$ II RT enzyme (Invitrogen) as described in [29]. The transcripts were analyzed by quantitative real-time PCR (ABI PRISM ${ }^{\mathrm{TM}} 7000$, Applied Biosystems, http://www.appliedbiosystems.com which was conducted using the $\mathrm{qPCR}^{\mathrm{TM}}$ core reagent kit (Eurogentec, http://www.eurogentec.com). To analyze Nadefensin, a specific TaqMan primer pair (forward primer: 5'-AACTATGGCTCG CTCCTTGTGC-3', the reverse primer: 5'CTCATAGGCAACAAAAAGCAT-3') and a double fluorescent dye-labeled probe (5'-TTCATGGCATTTGCAGTCTTGGCAA-3') were used. The relative gene expression was calculated using a 10-fold dilution series of CDNAs which had been transcribed from induced RNA samples from the same experiment.

\section{Analysis of herbivory}

Leaf area damage and larval mass

We placed 5-day-old larvae that were previously reared on WT N. attenuata leaves individually on the fully developed leaves of rosette-stage WT and irdefensin lines (76 and 96) that were either pre-infected with PST DC3000 or left uninfected $(\mathrm{N}=30)$. Each larva was enclosed in a well-aerated $5 \mathrm{~cm}$ diameter clip cage. The larvae were weighed 6 , 9 and 12 days after feeding. Leaf area damage was estimated at the end of 12 days and based on the extent of leaf damage, with values ranging from 1 to $5[1(0-15 \%)$, $2(16-30 \%), 3(31-45 \%), 4(46-60 \%), 5(61-75 \%)$ and $7(>76 \%)]$ was assigned.

\section{Analysis of pathogen growth}

To quantify the disease spread in WT and irdefensin lines (76 and 96) plants, we quantified the colony forming units (CFUs) in PST DC3000 inoculated leaves. In brief, surface-sterilized leaf discs $\left(1 \mathrm{~cm}^{2}\right)$ were ground in $1 \mathrm{ml}$ sterile water and $40 \mu \mathrm{l}$ of supernatant was spread on plates containing LB agar containing rifamycin and tetracycline. Colonies were counted after $48 \mathrm{~h}$ of incubation at $28^{\circ} \mathrm{C}$.

\section{Statistical analysis}

Data were analyzed with StatView (Abacus Concepts, Inc., http://findarticles.com/). 


\section{Authors' contributions}

CR carried out the molecular studies, herbivore and pathogen bio-assays and statistical analysis. ITB designed and coordinated the experiments and also helped to draft the manuscript. CR and ITB read and approved the final manuscript

\section{Acknowledgements}

We thank Dr. Klaus Gase, Thomas Hahn, Susan Kutschbach, Antje Wissgott, and Wibke Kroeber for assistance in preparing, cloning, sequencing analysis, and bacterial inoculations; Emily Wheeler for editorial assistance; and the Max Planck Society for funding.

\section{References}

I. Stout MJ, Fidantsef AL, Duffey SS, Bostock RM: Signal interactions in pathogen and insect attack: systemic plant-mediated interactions between pathogens and herbivores of the tomato, Lycopersicon esculentum. Physiological and Molecular Plant Pathology 1999, 54:1 I5-130.

2. Baldwin IT: Inducible nicotine production in native Nicotiana as an example of adaptive phenotypic plasticity. Journal of Chemical Ecology 1999, 25:3-30.

3. Steppuhn A, Gase K, Krock B, Halitschke R, Baldwin IT: Nicotine's defensive function in nature. Plos Biology 2004, 2: 1074-1080.

4. Keinanen M, Oldham NJ, Baldwin IT: Rapid HPLC screening of jasmonate-induced increases in tobacco alkaloids, phenolics, and diterpene glycosides in Nicotiana attenuata. Journal of Agricultural and Food Chemistry 200I, 49:3553-3558.

5. Van Dam NM, Horn M, Mares M, Baldwin IT: Ontogeny constrains systemic protease inhibitor response in Nicotiana attenuata. Journal of Chemical Ecology 200 I, 27:547-568.

6. Zavala JA, Patankar AG, Gase K, Hui DQ, Baldwin IT: Manipulation of endogenous trypsin proteinase inhibitor production in Nicotiana attenuata demonstrates their function as antiherbivore defenses. Plant Physiology 2004, 134:1 181-1190.

7. van Loon LC, Rep M, Pieterse CMJ: Significance of inducible defense-related proteins in infected plants. Annual Review of Phytopathology 2006, 44: 135-162.

8. Grover A, Gowthaman R: Strategies for development of fungusresistant transgenic plants. Current Science 2003, 84:330-340 [http://www.iisc.ernet.in/currsci/feb/02003/330.pdf].

9. Rayapuram C, Wu J, Haas C, Baldwin IT: PR-13/thionin not PR-I mediates bacterial resistance in Nicotiana attenuata in nature and neither influences herbivore resistance. Molecular Plant Microbe Interactions 2008, 21:988-100.

10. Wilding N, Collins M, Hammond PM, Webber JF: Insect-fungus interactions. Royal Entomological Society Symposium Series eds 1989 [http://openlibrary.org/b/OLI I45074M]. Academy press London

II. Biere A, Marak HB, van Damme JMM: Plant chemical defense against herbivores and pathogens: generalized defense or trade-offs? Oecologia 2004, I40:430-44I.

12. Krischik VA: Specific or generalized plant defense: reciprocal interactions between herbivores and pathogens. Microbial mediation of plant herbivore interactions 1991:309-340 [http:/l eu.wiley.com/WileyCDA/WileyTitle/productCd-047/6I324X.html]. New York, Academic Press

13. De Vos M, Van Zaanen W, Koornneef A, Korzelius JP, Dicke M, Van Loon LC, Pieterse CMJ: Herbivore-induced resistance against microbial pathogens in Arabidopsis. Plant Physiology 2006, I42:352-363.

14. Lou Y, Baldwin IT: Nitrogen supply influences herbivoreinduced direct and indirect defenses and transcriptional responses to Nicotiana attenuata. Plant Physiology 2004, I35:496-506.

15. Voelckel C, Baldwin IT: Herbivore-induced plant vaccination. Part II. Array-studies reveal the transience of herbivore-specific transcriptional imprints and a distinct imprint from stress combinations. Plant Journal 2004, 38:650-663.

16. Heidel AJ, Baldwin IT: Microarray analysis of salicylic acid- and jasmonic acid-signaling in responses of Nicotiana attenuat a to attack by insects from multiple feeding guilds. Plant Cell and Environment 2004, 27:1362-1373.
17. Carmona MJ, Molina A, Fernandez JA, Lopezfando J], Garciaolmedo F: Expression of the alpha-thionin gene from barley in tobacco confers enhanced resistance to bacterial pathogens. Plant Journal I993, 3:457-462

18. Florack DEA, Visser B, Devries PM, Vanvuurde JWL, Stiekema WJ: Analysis of the toxicity of purothionins and hordothionins for plant-pathogenic bacteria. Netherlands Journal of Plant Pathology 1993, 99:259-268.

19. Bohlmann H, Vignutelli A, Hilpert B, Miersch O, Wasternack C, Apel $\mathrm{K}$ : Wounding and chemicals induce expression of the Arabidopsis thaliana gene Thi2.I, encoding a fungal defense defensin, via the octadecanoid pathway. FEBS Letters 1998 437:28I-286

20. Cui JP, Jander G, Racki LR, Kim PD, Pierce NE, Ausubel FM: Signals involved in Arabidopsis resistance to Trichoplusia ni caterpillars induced by virulent and avirulent strains of the phytopathogen Pseudomonas syringae. Plant Physiology 2002, I 29:55I-564.

21. Stotz HU, Koch T, Biedermann A, Weniger K, Boland W, MitchellOlds T: Evidence for regulation of resistance in Arabidopsis to Egyptian cotton worm by salicylic and jasmonic acid signaling pathways. Planta 2002, 21 4:648-652.

22. Rayapuram C, Baldwin IT: Increased SA in NPR I-silenced plants antagonizes JA and JA-dependent direct and indirect defenses in herbivore-attacked Nicotiana attenuata in nature. Plant Journal 2007, 52:700-7I5.

23. Stec B: Plant thionin - the structural perspective. Cellular and Molecular Life Sciences 2006, 63: 1370-1385.

24. Karban R, Adamchak R, Schnathorst WC: Induced resistance and interspecific competition between spider-mites and a vascular wilt fungus. Science 1987, 235:678-680.

25. Hatcher PE, Paul ND: Beetle grazing reduces natural infection of Rumex obtusifolius by fungal pathogens. New Phytologist 2000 , I 46:325-333.

26. Baldwin IT: The alkaloidal responses of wild tobacco to real and simulated herbivory. Oecologia 1988, 77:378-381.

27. Kruegel T, Lim M, Gase K, Halitschke R, Baldwin IT: Agrobacterium-mediated transformation of Nicotiana attenuata, a model ecological expression system. Chemoecology 2002, I 2:177-183.

28. Katagiri F, Thilmony R, He SY: The Arabidopsis Thaliana-Pseudomonas Syringae Interaction. The Arabidopsis Book [http:// www.aspb.org/publications/arabidopsis/]. Rockville, MD, American Society of Plant Biologists

29. Rayapuram C, Baldwin IT: Using nutritional indices to study LOX3-dependent insect resistance. Plant Cell and Environment 2006, 29:1585-1594.

Publish with Bio Med Central and every scientist can read your work free of charge

"BioMed Central will be the most significant development for disseminating the results of biomedical research in our lifetime. "

Sir Paul Nurse, Cancer Research UK

Your research papers will be:

- available free of charge to the entire biomedical community

- peer reviewed and published immediately upon acceptance

- cited in PubMed and archived on PubMed Central

- yours - you keep the copyright
BioMedcentral 